1 Faculdade de Tecnologia das Ciências (FTC) Vitória da Conquista (BA), Brasil. Faculdade Independente do Nordeste (Fainor) - Vitória da Conquista (BA), Brasil. vnessa.cruz@yahoo.com.br

2 Faculdade Independente do Nordeste (Fainor) -

Vitória da Conquista (BA), Brasil.

lucianareisfainor@gmail.com

3 Faculdade Independente do Nordeste (Fainor) -

Vitória da Conquista (BA),

Brasil.

karlinhakau@hotmail.com

4 Faculdade Independente do Nordeste (Fainor) -

Vitória da Conquista (BA), Brasil.

juliananbarros@fainor.com.br

5 Prefeitura de Vitória da Conquista, Diretoria de Atenção Programática e Especializada - Vitória da conquista (BA), Brasil. tarcycastro@yahoo.com.br

\section{Percepção da mãe ou cuidador de crianças asmáticas sobre os resultados do tratamento}

\author{
Perception of mothers or caregivers of children with asthma on \\ treatment outcomes
}

Vanessa Cruz Miranda', Luciana Araújo dos Reis $\mathbf{2}$, Karla Cavalcante Silva de Morais $\mathbf{3}$, Juliana Barros Ferreira ${ }^{4}$, Tarcísia Castro Alves ${ }^{5}$

RESUMO A percepção da mãe ou cuidador em relação aos resultados alcançados é um instrumento de grande relevância para identificar o grau de resolutividade do serviço e garantir uma maior qualidade da assistência. Nessa perspectiva, foi realizada pesquisa descritiva com abordagem quantitativa a partir da aplicação de questionário. O estudo objetivou avaliar a percepção da mãe ou cuidador em relação ao resultado do tratamento que a criança asmática recebe. Concluiu-se que um maior percentual de mães ou cuidadores consideraram o resultado como bom/muito bom e ruim/péssimo, além de identificar que essas crianças são acompanhadas apenas pelo pneumologista, e não pela equipe da unidade de saúde.

PALAVRAS-ChAVe Asma. Gestão da qualidade. Sistema Único de Saúde. Percepção.

\begin{abstract}
The perception of the mother or caregiver regarding the achieved results is a very important tool to identify the degree of resoluteness of the service and ensure a higher quality of care. In that perspective, a descriptive research with quantita-tive approach from the application of a questionnaire was carried out. The study aimed to evaluate the perception of the mother or caregiver regarding the outcome of the treatment that the asthmatic child receives. It was concluded that a higher percentage of mothers or caregivers considered the result as good/very good and bad/very bad, in addition to identifying that these children are accompanied only by a pulmonologist and not by the health unit staff.
\end{abstract}

KEYWORDS Asthma. Quality management. Unified Health System. Perception. 


\section{Introdução}

A asma é uma das afecções crônicas mais comuns em crianças, contribuindo com grande parcela das causas de morbimortalidade (BRASIL, 2010). O Brasil ocupa a oitava posição mundial em prevalência de asma, com aproximadamente 20 milhões de asmáticos, que, dependendo da região e da faixa etária, varia de menos que $10 \%$ a mais do que $20 \%$ em diversas cidades estudadas (BRASIL, 2010). Em 2011, foi a quarta causa de internações, registrando 160 mil hospitalizações (SBPT, 2012).

É um problema mundial de saúde pública que compromete a população infantil devido a sua alta morbimortalidade, absenteísmo na escola, causando, portanto, grande impacto econômico, social e emocional (BRASIL, 2010). Essas limitações físicas, intelectuais e emocionais, como consequência da doença, levam ao sofrimento humano por parte do paciente e dos familiares, uma vez que comprometem o curso natural da vida deste e a sua qualidade de vida (BRASIL, 2010).

Nesse contexto, profissionais de saúde (médicos), Governo e organismos internacionais de saúde identificaram a necessidade de buscar uma maior compreensão sobre o tratamento dessa doença, em que houvesse a troca de informações e de experiências entre profissionais de saúde de diversos países, para que, assim, pudessem oferecer ao asmático um atendimento mais adequado e completo (FONTES ET AL., 2011). Para tanto, em 1995, foi criado pela Organização Mundial da Saúde (OMS) o programa chamado Iniciativa Global da Asma (Gina) que vigora até os dias atuais. Esse programa trouxe como propostas: um maior reconhecimento da asma por parte dos profissionais de saúde e da população; a priorização da prevenção, o diagnóstico e controle da doença; a educação da população com relação à prevenção e controle e estímulo à pesquisa acerca da patologia. Dessa forma, o Gina pretendeu estabelecer uma abordagem integral à saúde dessa população e promover o vínculo entre o paciente e a unidade de saúde (FONTES ET AL., 2011).

A partir de então, foram realizados encontros nacionais e internacionais com o objetivo de estabelecer rotinas diagnósticas e terapêuticas para a doença, sendo o Brasil influenciado por essas discussões. Em 1996, foi elaborado o I Consenso Brasileiro de Educação em Asma, que estabeleceu as bases para a organização e implantação de um programa de educação adaptado às condições sociais, econômicas e culturais para o tratamento da população em questão. O II Consenso Brasileiro no Manejo da Asma, em 1998, definiu a doença, a sua fisiopatologia e o seu tratamento (AMARAL; PALMA; LEITE, 2012).

O III Consenso Brasileiro no Manejo da Asma definiu os princípios de manutenção do controle da doença. Tais princípios buscam orientar os pacientes com asma de forma individual ou em grupo sobre sua doença; informar sobre como eliminar ou controlar os fatores desencadeantes, bem como sobre o tratamento em casos graves. Diante disso e das propostas do guia para 'Manejo da rinite alérgica e seu impacto na asma', em 2004, o Ministério da Saúde publicou o manual técnico 'Asma e rinite: linhas de conduta em atenção básica', que inclui o tratamento da asma leve e moderada na rede de atenção básica (AMARAL; PALMA; LEITE, 2012; SOUZA; MARCH, 2010).

Em seguida, foram lançadas as 'IV Diretrizes brasileiras para o manejo da asma', que se iniciam com a etiopatogenia e epidemiologia, depois versam sobre a classificação clínica da gravidade e controle, o tratamento da manutenção e da crise, a educação em asma e, por fim, fornecem as informações legais referentes ao subsídio dos medicamentos que estão disponíveis no País (SOUZA; MARCH, 2010). Em 2012, foi publicado o livro que contém as 'Diretrizes da Sociedade Brasileira de Pneumologia e Tisiologia para o manejo da asma' a partir da opinião de especialistas pneumologistas e pneumopediatras 
e das recomendações do Gina, do National Asthma Education and Prevention Program (Naepp), dos consensos britânico e canadense para tratamento da asma. O referido livro apresenta o manejo da doença para todas as idades e aponta a educação em asma como um fator de grande relevância para garantir melhor qualidade de vida e modificação dos indicadores de saúde (BATISTA, 2013).

Segundo o Gina (2010), apesar de todo esforço, os resultados, de modo geral, ainda não são satisfatórios, pois, mesmo nos locais onde programas foram implantados, existem dificuldades na mudança de comportamento, de forma permanente, conforme preconizam os consensos e diretrizes. Segundo Kinchoku et al. (2011), os fatores importantes para o baixo percentual de controle incluem o uso inadequado das medicações, os baixos índices de diagnóstico, tratamento inadequado e a falta de monitoramento.

Assim, é de fundamental importância avaliar a assistência que vem sendo prestada à população pediátrica asmática e os resultados alcançados sob a perspectiva da mãe ou cuidador, a fim de identificar os possíveis entraves existentes e garantir a qualidade do serviço e atender as expectativas da população.

A percepção do usuário sobre determinado serviço é individual e pode ser influenciada por alguns fatores, como: resolutividade; atendimento das demandas de saúde da população; satisfação do usuário; tecnologias oferecidas; existência de um sistema de referência e contrarreferência preestabelecido; acessibilidade dos serviços; adesão ao tratamento; aspectos culturais e socioeconômicos da comunidade. Entraves em um ou mais desses fatores podem influenciar negativamente a percepção do indivíduo sobre o serviço oferecido. Sabe-se que uma das maneiras de avaliar a qualidade e efetividade do serviço é a partir da resolutividade. Acredita-se, portanto, que quanto maior a resolutividade de um serviço, melhor são atendidas as demandas de saúde da população e maior será sua satisfação (TURRINI; LEBRÃO; CESAR, 2008).

A partir desse pressuposto, o presente estudo, teve como objetivo avaliar a percepção da mãe ou cuidador em relação ao resultado do tratamento que a criança asmática recebe.

\section{Métodos}

Trata-se de uma pesquisa exploratória e descritiva seguindo o delineamento transversal, com abordagem quantitativa.

Os locais de estudo foram duas Unidades Básicas de Saúde da área urbana do município de Vitória da Conquista que possuíam Estratégia Saúde da Família (ESF). O sorteio dessas unidades foi feito por conveniência entre as 14 unidades que possuem ESF no município. A coleta de dados foi realizada na casa das mães ou cuidadores de crianças asmáticas cadastradas nessas duas unidades.

A amostra foi composta por mães ou cuidadores de crianças asmáticas com idade inferior a 12 anos incompletos, já que o conceito de criança utilizado foi o do Estatuto da Criança e do Adolescente (ECA). Além disso, as mães ou cuidadores deveriam ter idade superior a 18 anos, e as crianças asmáticas, no mínimo, 1 ano de assistência na unidade pesquisada. Os critérios de exclusão foram: mães ou cuidadores de crianças asmáticas que não se encontrassem em suas residências após três tentativas; mães ou cuidadores que não tivessem sido reconhecidas pelas enfermeiras ou agentes de saúde.

Ao final desse levantamento, a pesquisadora possuía um total de 75 nomes de mães ou cuidadores, sendo uma lista com 33 nomes fornecida pelas enfermeiras responsáveis e a outra com 42 nomes fornecida pela Central de Regulação de marcação de exames da prefeitura. Dessas listas, duas mães recusaram 
participar; uma não possuía um ano de cadastro na unidade; quatro não moravam mais no bairro; 16 não foram reconhecidas pelas agentes comunitárias e enfermeiras, uma não era asmática e uma tinha idade superior a 12 anos incompletos. Por fim, a amostra do estudo foi composta por 50 mães ou cuidadores, sendo 24 da unidade de saúde I e 26 da unidade de saúde II.

O cálculo amostral não foi realizado, uma vez que as unidades de saúde não possuíam registros sobre as crianças asmáticas e o centro de procedimentos de dados da Secretaria de Saúde do Município não possuía nenhum registro referente à incidência, prevalência da doença ou de internações.

O instrumento foi composto por um questionário elaborado pela pesquisadora, sendo utilizado como base o PCATool-Brasil (Primary Care Assessment Tool-Brasil) versão criança. O questionário foi aplicado às mães ou cuidadores de crianças asmáticas, atendidas na atenção básica, com objetivo de identificar a percepção da mãe ou cuidador sobre o tratamento oferecido às crianças asmáticas.

O questionário possuía um total de 18 perguntas e foi subdividido em 2 tópicos: dados sociodemográficos (caracterização das crianças, mães ou cuidadores e dados socioeconômicos) e avaliação da assistência.

O tópico referente aos dados sociodemográficos foi composto por nove questões. Teve como objetivo caracterizar a amostra quanto às informações de identificação da criança e identificação da mãe ou cuidador e renda familiar.

O tópico Avaliação da assistência foi constituído por dez questões. Teve como objetivo avaliar a percepção da mãe ou cuidador levando em consideração a longitudinalidade da assistência e os resultados do tratamento oferecido às crianças asmáticas. Sendo as respostas 'Sim' ou 'Não' para as seguintes indagações: médico/enfermeiro acompanha a criança; sente-se à vontade de conversar com o profissional; médico/enfermeiro tira dúvida e você entende; médico/enfermeiro conhece história clínica completa da criança; médico sabe todos os medicamentos que a criança usa, diagnóstico precoce; internação frequente. Para as perguntas quanto ao resultado do tratamento, foram utilizadas as respostas: 'muito bom, bom, regular, ruim, péssimo’.

De posse dos resultados, os dados da pesquisa foram inseridos em uma planilha do Programa Excel ${ }^{\circledR} 2015$ e posteriormente transportados para o Programa Statistical Pack age for the Social Science SPSS ${ }^{\circledR}$ versão 20.0, no qual foi realizada a análise estatística descritiva, sendo calculada frequência absoluta e relativa, média e desvio padrão.

$\mathrm{O}$ projeto de pesquisa foi submetido e aprovado pelo Comitê de Ética em Pesquisa da Fundação Oswaldo Cruz (Fiocruz), parecer número 1.145.709. Os participantes da pesquisa assinaram o Termo de Consentimento Livre e Esclarecido. Foi utilizado também o Termo de Compromisso de Uso de Dados (TCUD).

\section{Resultados e discussão}

Para melhor entendimento, os resultados foram divididos em quatro grupos: (1) Caracterização das crianças; (2) Caracterização das mães ou cuidadores (3); Dados socioeconômicos; (4) Avaliação da assistência.

\section{(1) Caracterização das crianças}

Houve um maior predomínio de crianças do sexo masculino $(60,0 \%)$, na faixa etária entre 4 e 8 anos $(40,0 \%)$ e que frequentavam a escola pública (34,0\%). Segundo dados da tabela 1. 
Tabela 1. Caracterização da criança com relação ao sexo, faixa etária, educação creche/escola. Vitória da Conquista (BA), 2015

\begin{tabular}{lccc}
\hline & & $\mathbf{N}$ & $\%$ \\
\hline Sexo & Masculino & 30 & 60,0 \\
& Feminino & 20 & 40,0 \\
\hline Faixa etária & 1 a 3 anos & 18 & 36,0 \\
& 4 a 8 anos & 9 a 13 anos & 40,0 \\
& Escola pública & 12 & 24,0 \\
\hline Escola/Creche & Escola particular & 17 & 34,0 \\
& Creche pública & 50,0 \\
& Creche particular & 5 & 10,0 \\
& Não frequenta & 1 & 2,0 \\
\hline
\end{tabular}

Fonte: Elaboração própria.

No que concerne à caracterização das crianças asmáticas, o sexo masculino foi prevalente, corroborando o estudo realizado a partir de dados secundários oriundos da Pesquisa Nacional por Amostra de Domicílios (PNAD) e seu suplemento sobre saúde de crianças da região Nordeste e Sudeste, Norte e Centro-Oeste. Este teve como finalidade a produção de informações básicas para o estudo do desenvolvimento socioeconômico do País (WeHRMEISTER; PERES, 2010).

Telles Filho (2012) explica esse predomínio da asma em crianças do sexo masculino devido às vias aéreas mais estreitas; a maior produção de IgE (imunoglobulina) e a maior contração das vias aéreas.

Verificou-se também que, nesse estudo, houve um maior predomínio de crianças na faixa etária entre 4 e 8 anos, sendo esta considerada de risco para internações. Denota-se a necessidade de uma assistência adequada incluindo prevenção e controle para reduzir de maneira efetiva esses indicadores. Esse dado é confirmado em estudo realizado a partir de dados secundários oriundos da PNAD e seu suplemento sobre saúde de crianças da região Nordeste e Sudeste, Norte e Centro-Oeste, no qual os resultados apontaram para uma maior prevalência de internação na faixa etária entre 3 e 7 anos, tanto na região Nordeste quanto na região Sul (WEHRMEISTER; PERES, 2010).

Em pesquisa realizada no município de Salvador (BA) com análise do tipo ecológico de agregados de séries temporais, a partir de dados de internações hospitalares, observou-se que as maiores taxas de asmáticos ocorrem na faixa etária de menores de 15 anos. Segundo o autor, apesar desse acometimento ser comum na infância, as internações podem ser evitadas quando os familiares são adequadamente orientados sobre a doença e têm acesso aos cuidados necessários (CARDOSO; OLIVEIRA, 2011). 


\section{(2) Caracterização das mães ou cuidadores}

Conforme dados da tabela 2, observou-se uma maior frequência de mães ou cuidadores com ensino fundamental incompleto $(34,0 \%)$, com trabalho fixo $(54,0 \%)$ e com carga horária semanal de trabalho de 20 a 40 horas (28,0\%). A média de idade das mães ou cuidadores foi de $34,24( \pm 8,16)$ anos.

Tabela 2. Caracterização da mãe ou cuidador com relação ao grau de escolaridade, trabalho fixo e carga horária semanal trabalhada. Vitória da Conquista (BA), 2015

\begin{tabular}{|c|c|c|c|}
\hline & & $\mathbf{N}$ & $\%$ \\
\hline \multirow{6}{*}{ Escolaridade } & Não respondeu & 1 & 2,0 \\
\hline & Ensino Fundamental Incompleto & 17 & 34,0 \\
\hline & Ensino Fundamental & 8 & 16,0 \\
\hline & Ensino Médio Incompleto & 8 & 16,0 \\
\hline & Ensino Médio & 15 & 30,0 \\
\hline & Ensino Superior & 1 & 2,0 \\
\hline \multirow{2}{*}{ Trabalho fixo } & Sim & 23 & 46,0 \\
\hline & Não & 27 & 54,0 \\
\hline \multirow{4}{*}{ Carga horária } & Não trabalha & 27 & 54,0 \\
\hline & Menos de 30 horas & 2 & 4,0 \\
\hline & 20 a 40 horas & 14 & 280 \\
\hline & 41 a 60 horas & 7 & 14,0 \\
\hline Total & & 50 & $100, c$ \\
\hline
\end{tabular}

Fonte: Elaboração própria.

Constatou-se no presente estudo que a maioria das mães possui como nível de escolaridade o ensino fundamental incompleto, sendo este dado preocupante já que o grau de escolaridade dos pais pode influenciar no cuidado da criança asmática. Confirma-se essa informação em estudo caso-controle, retrospectivo-observacional, com amostra de 138 crianças que verificou que quanto menor o nível de escolaridade e informação das mães, maior ocorrência de asma e menor reconhecimento dos sintomas apresentados pelos filhos. Esses sintomas podem ser característicos de um episódio leve de asma e ser ignorado, podendo provocar o agravamento da doença (ROCHA ET AL., 2011).

Conforme Perosa et al. (2013) em estudo transversal com aplicação de questionário de Qualidade de Vida (QV) a 42 crianças e escala de enfrentamento aplicado às mães, verificaram que a escolaridade do cuidador mostrou-se preditora de melhor índice de QV, uma vez que a alta escolaridade materna tem sido associada com a capacidade de cuidar adequadamente, com a maior valorização do conhecimento científico e com a capacidade de articular recursos em prol das necessidades da criança. Mães com 
mais escolaridade têm mais conhecimentos quanto à asma e sua evolução, sobre o que fazer quando os sintomas aparecem e como prevenir as crises, minimizando os prejuízos à QV da criança.

Quanto ao presente trabalho, verificou-se que houve uma maior frequência de mães donas de casa, fato que favorece uma maior disponibilidade de tempo destas para um melhor cuidado com a criança. Coriolano (2011), em seu estudo, observou que mães que trabalham exclusivamente no domicílio têm maior possibilidade de implementar cuidados que favorecem a prevenção de doenças.

\section{(3) Dados socioeconômicos}

A renda familiar mensal total das mães ou cuidadores pesquisados foi de $64,0 \%$ possuindo de 1 a 3 salários mínimos. Com relação à quantidade de pessoas que contribuíam para a renda mensal, somente uma pessoa contribuía em 56,0\% dos casos. Quanto ao número de pessoas que eram sustentadas por essa renda, $44,0 \%$ sustentavam quatro pessoas. Conforme tabela 3 .

Tabela 3. Renda familiar: analisando a renda mensal total, número de pessoas que contribuem para obtenção da renda e quantos sustentados pela renda. Vitória da Conquista (BA), 2015

\begin{tabular}{|c|c|c|c|}
\hline & & $\mathbf{N}$ & $\%$ \\
\hline & Até 1 salário mínmo & 17 & 34,0 \\
\hline \multirow[t]{3}{*}{ Renda mensal total } & Mais de 1 até 3 salários mínimos & 32 & 64,0 \\
\hline & Mais de 3 até 5 salários mínimos & 1 & 2,0 \\
\hline & Uma & 28 & 56,0 \\
\hline \multirow[t]{4}{*}{ Número de pessoas que contribuem } & Duas & 20 & 40,0 \\
\hline & Três & 2 & 4,0 \\
\hline & Três & 8 & 16,0 \\
\hline & Quatro & 22 & 44,0 \\
\hline \multirow[t]{2}{*}{ Sustentados pela renda } & Cinco & 9 & 18,0 \\
\hline & Mais de cinco & 11 & 22,0 \\
\hline Total & & 50 & 100,0 \\
\hline
\end{tabular}

Fonte: Elaboração própria.

Em estudo realizado com um total de 6.437 escolares, sendo 3.183 com faixa etária de 6 a 7 anos e 3.254 de 13 a 14 anos, foi aplicado o questionário Isaac (International Study of Asthma and Allergies in Childhood) (fase 3) em escolas públicas e particulares do Distrito Federal, que tinha como objetivo avaliar a prevalência de asma brônquica, os sintomas a ela relacionados e sua relação com o nível socioeconômico. Foi verificado que, quanto menos favorecida a classe social, maiores são as prevalências de diagnóstico de asma, dos sintomas a ela relacionados e maior número de crises. Segundo o autor, isso se deve à influência de fatores ambientais, como ambiente domiciliar com aglomeração de pessoas; maior exposição a mofo; poeira e pelos de animais; tabagismo passivo 
e dificuldade de acesso a um tratamento adequado para a doença (FELIZOLA ET AL., 2005).

Wehrmeister e Peres (2010) corroboram o autor supracitado e afirmam que os efeitos da renda sobre a saúde dos indivíduos estão associados à possibilidade do consumo de bens que proveem ambientes favoráveis à saúde, o que acaba por ferir o princípio constitucional de equidade.

A renda da família é um fator que influencia na prevalência de asma e no acesso a serviços e bens de consumo favorecendo a saúde do indivíduo. Na atual pesquisa, a grande maioria dos pesquisados possuía uma renda mensal total de mais de 1 até 3 salários mínimos, sendo, portanto classificados conforme a tabela de classe social do Instituto Brasileiro de Geografia e Estatística (IBGE) como grupo D e E. Estes grupos são desfavorecidos, pois apresentam menor potencial para o consumo de bens.

\section{(4) Avaliação da assistência}

No que diz respeito ao atributo longitudinalidade, observou-se que $82,0 \%$ das crianças asmáticas não possuíam médico/ enfermeiro que as acompanhassem em seu problema. Quando perguntado se essas mães ou cuidadores se sentiam à vontade de conversar com o profissional que atende à criança, 70,0\% relataram que sim. Quanto à disponibilidade dos médicos/ enfermeiros na escuta das dúvidas e nas explicações destas, $58,0 \%$ das mães ou cuidadores referiram que os médicos/ enfermeiros escutavam suas dúvidas e explicavam de forma que elas entendem. Segundo relato das mães ou cuidadores, $68,0 \%$ dos médicos/enfermeiros não conheciam a história clínica completa da criança, e 74,0\% dos médicos não sabiam todos os medicamentos que a criança fazia uso (tabela 4).

Tabela 4. Avaliação do serviço prestado pela unidade de saúde da família às crianças asmáticas quanto ao atributo longitudinalidade. Vitória da Conquista (BA), 2015

\begin{tabular}{|c|c|c|c|}
\hline & & $\mathbf{N}$ & $\%$ \\
\hline \multirow{2}{*}{ Médico/enfermeiro da unidade acompanha criança } & $\operatorname{Sim}$ & 9 & 18,0 \\
\hline & Não & 41 & 82,0 \\
\hline \multirow{3}{*}{ Sente-se a vontade de conversar com o profissional } & Não respondeu & 2 & 4,0 \\
\hline & $\operatorname{sim}$ & 35 & 70,0 \\
\hline & Não & 13 & 26,0 \\
\hline \multirow{3}{*}{ O médico/enfermeiro tira sua dúvida e você entende } & Não respondeu & 5 & 10,0 \\
\hline & $\operatorname{Sim}$ & 29 & 58,0 \\
\hline & Não & 16 & 32,0 \\
\hline \multirow[b]{2}{*}{ O médico/enfermeiro conhece história clínica complet } & $\operatorname{Sim}$ & 16 & 32,0 \\
\hline & Não & 34 & 68,0 \\
\hline \multirow{2}{*}{ Médico sabe todos os medicamentos que a criança us } & $\operatorname{Sim}$ & 13 & 26,0 \\
\hline & Não & 37 & 74,0 \\
\hline Total & & 50 & 100,0 \\
\hline
\end{tabular}


Quanto ao atributo longitudinalidade, observou-se resultados negativos em algumas dimensões, pois a maior parte das crianças não possuía médico/enfermeiro na unidade que as acompanhassem, a maioria dos médicos/enfermeiros não conhecia a história clínica completa da criança e boa parte não sabia todos os medicamentos que a criança fazia uso. O ponto positivo desse atributo esteve relacionado com os quesitos: se o entrevistado se sente à vontade para conversar com o profissional de saúde e quanto à escuta do médico/enfermeiro sobre suas dúvidas e explicação de forma que ele entenda; verificou-se uma maior distribuição de mães que responderam sim aos quesitos.

O termo 'longitudinalidade' é pouco utilizado no Brasil, logo a definição utilizada nesta pesquisa foi a de Starfield, que conceitua a longitudinalidade como a inter-relação de longa duração entre profissionais de saúde e pacientes nas suas unidades de saúde, mediada por laços interpessoais que, consequentemente, reflete em mútua cooperação entre os usuários e os profissionais de saúde (CUNHA; GIOVANELLA, 2011).

Baratieri e Marcon (2011) também utilizaram o conceito de longitudinalidade de Starfield em seu estudo descritivo-exploratório com abordagem qualitativa, que teve como objetivos: avaliar como os enfermeiros conceituam a longitudinalidade do cuidado na ESF e verificar sua compreensão sobre a efetivação dessas em seu trabalho e com a equipe. A partir dessa pesquisa, o autor pôde perceber que a garantia da longitudinalidade da atenção necessita de uma boa interação entre as partes, confiança e responsabilidade ao longo de toda a relação, sendo essa característica, de fonte regular de cuidado ao longo do tempo, o ponto central para uma maior efetividade da atenção em saúde.

Percebe-se que muitas equipes de ESF não assumem ou não estabelecem em sua rotina a coordenação das informações pregressas acerca da histórica clínica dos usuários, a referência e a contrarreferência referentes ao grupo de sujeitos que acessam o serviço regularmente, dificultando assim o estabelecimento da relação de confiança mútua (SANTOS; GIOVANELLA, 2016).

É importante citar sobre o uso da palavra continuidade como sinônimo de longitudinalidade, entretanto estas palavras possuem conceitos distintos. A continuidade do cuidado é definida como o acompanhamento de uma patologia específica do paciente, porém não obrigatoriamente por um mesmo médico. A continuidade não exige inter-relação entre profissional e usuário, já que bons registros podem substituir a necessidade de informação para o devido acompanhamento da patologia (CUNHA; GIOVANELLA, 2011; BARATIERI; MARCON, 2011).

Em pesquisa qualitativa exploratória realizada com trabalhadores de saúde e usuários cadastrados na unidade de saúde, foi observado que o médico/enfermeiro que já conhecia a história dos usuários era preferido por eles para o acompanhamento longitudinal. Dessa forma, era mais provável a realização de encaminhamentos quando necessário; diagnóstico mais eficaz; reconhecimento dos problemas e necessidades dos usuários, melhor escolha de tratamento medicamentoso; melhor orientação em relação ao tratamento e consequentemente maior satisfação do usuário em relação ao seu cuidado (GHIGGl; BARRETO; FAJARDO, 2014).

Os usuários que possuem uma unidade ou um profissional como sua fonte habitual de cuidado mantêm suas consultas de revisão e conseguem se prevenir quanto aos problemas de saúde, assim como pacientes que procuram sempre o mesmo local como fonte de cuidado possuem menores índices de hospitalização e menor custo do tratamento quando comparado àqueles que não possuem esse vínculo. Dessa forma, se o usuário for sempre acompanhado pelo mesmo profissional, essa redução é ainda maior (GHIGGI; BARRETO; FAJARDO, 2014).

Os resultados encontrados quanto ao 
atributo longitudinalidade demonstram o quanto a asma é pouco priorizada pelos serviços como uma doença crônica grave, que necessita de tratamento contínuo e integral, acompanhamento e desenvolvimento de processo educacional e percepções sobre a doença. É necessário, portanto, uma assistência sem atrasos no diagnóstico; acessível à população; com profissionais sensibilizados e capacitados; medicação de primeira linha e investimento em educação em saúde. Isso requer um investimento certamente inferior aos custos das internações, do absenteísmo escolar e um menor sofrimento dessa população.

A tabela 5 aborda a avaliação dos resultados do tratamento dado às crianças asmáticas. Segundo a opinião das mães ou cuidadores, constatou-se que $68,0 \%$ das crianças tiveram diagnóstico precoce para asma. No que diz respeito à internação, 88,0\% não internavam com frequência, e 52,0\% não faltavam muito à escola/creche por causa da doença. Quanto ao resultado do tratamento que a criança recebe, $38 \%$ consideraram como muito bom/ bom e $34 \%$ ruim/péssimo.

Tabela 5. Avaliação dos resultados do tratamento dado às crianças asmáticas segundo a opinião das mães ou cuidadores. Vitória da Conquista (BA), 2015

\begin{tabular}{|c|c|c|c|}
\hline & & $\mathbf{N}$ & $\%$ \\
\hline \multirow{2}{*}{ Diagnóstico precoce } & Sim & 34 & 68,0 \\
\hline & Não & 16 & 32,0 \\
\hline \multirow{2}{*}{ Interna frequentemente } & Sim & 6 & 12,0 \\
\hline & Não & 44 & 88,0 \\
\hline \multirow{3}{*}{ Falta muito a escola } & Não frequenta & 3 & 6,0 \\
\hline & Sim & 21 & 42,0 \\
\hline & Não & 26 & 52,0 \\
\hline \multirow{5}{*}{ Resultado do tratamento } & Muito bom & 1 & 2,0 \\
\hline & Bom & 18 & 36,0 \\
\hline & Regular & 14 & 28,0 \\
\hline & Ruim & 6 & 12,0 \\
\hline & Péssimo & 11 & 22,0 \\
\hline Total & & 50 & 100,0 \\
\hline
\end{tabular}

Fonte: Elaboração própria.

Martins et al. (2014) realizaram estudo de revisão sistemática a partir de 30 artigos, tendo como objetivo analisar a percepção dos usuários e/ou dos profissionais quanto à qualidade dos serviços públicos de saúde.
Nesse estudo, foram registrados resultados abrangentes quanto à percepção dos usuários sobre a qualidade da atenção básica. Os pontos mais relevantes foram: facilidade para agendar consultas no serviço e no 
serviço especializado; tempo de espera; cordialidade no atendimento; boa relação profissional-usuário; resolução dos problemas e boas condições na estrutura física.

A partir desse contexto, é sabido que a qualidade e satisfação do usuário dependem dentre outros fatores da capacidade resolutiva do tratamento recebido, por isso foi pesquisada a opinião dos usuários quanto aos resultados alcançados. A maioria respondeu que as crianças tiveram diagnóstico precoce, mas que na maioria dos casos não esteve associado a um esforço da unidade, mas sim a partir do encaminhamento dos médicos do hospital para o especialista, que fez o diagnóstico após acompanhamento em consultas.

As crianças, em sua maior parte, não internam com frequência e não faltam muito à escola, o que, segundo as mães e ou cuidadores, só foi possível após acompanhamento da criança pelo pneumologista. Quanto ao resultado do tratamento recebido pela criança asmática, as mães ou cuidadores consideraram em sua maioria como muito bom/bom (38\%), porém foi encontrado um percentual de $34 \%$ que consideraram o tratamento como ruim/péssimo. Além disso, as entrevistadas foram unânimes em associar o resultado do tratamento como êxito dos pneumologistas que acompanham a criança, e não das unidades de saúde.

\section{Considerações finais}

A opinião das mães ou cuidadores quanto ao resultado do tratamento recebido pela criança asmática apresentou-se dividida, já que $38 \%$ consideraram como muito bom/ bom e $34 \%$ como ruim/péssimo. Fato que requer uma revisão de como vem sendo prestada a assistência a esse público.

Quanto aos resultados positivos alcançados no tratamento com relação à redução da hospitalização e absenteísmo escolar, as mães ou cuidadores direcionaram o êxito aos cuidados prestados pelo pneumologista que as acompanham, e não à assistência dada pela Unidade Básica de Saúde. Segundo as mães, as crianças asmáticas não são acompanhadas pela atenção primária, por isso os resultados não podem ser direcionados para esse setor.

Sugere-se que novos estudos sejam realizados com abordagem qualitativa para que, assim, uma análise subjetiva seja realizada, buscando apreender o entendimento das mães ou cuidadores com relação à assistência à saúde das crianças asmáticas recebida no município de Vitória da Conquista (BA). Propõe-se que a Secretaria de Saúde do Município crie um programa de assistência para essa população, definindo suas estratégias a partir de programas já existentes para o asmático, e qualifique os profissionais das unidades básicas para que o acompanhamento integral se concretize na prática.

\section{Colaboradores}

Vanessa Cruz Miranda: contribuiu substancialmente para a concepção, planejamento, análise, interpretação dos dados e participou da aprovação da versão final do manuscrito.

Luciana Araújo do Reis: contribuiu substancialmente para a concepção, planejamento, análise, interpretação dos dados e participou da aprovação da versão final do manuscrito.

Karla Cavalcante Silva de Moraes: contribuiu significativamente para revisão crítica do conteúdo e participou da aprovação da versão final do manuscrito.

Juliana Barros Ferreira: contribuiu significativamente para revisão crítica do conteúdo e participou da aprovação da versão final do manuscrito.

Tarcísia Castro Alves: contribuiu para análise, interpretação dos dados e participou da aprovação da versão final do manuscrito. 


\section{Referências}

AMARAL, L. M.; PALMA, P. V.; LEITE, I. C. G. Evolução das políticas públicas e programas de controle da asma no Brasil sob a perspectiva dos consensos. J bras pneumol., Brasília, DF, v. 38, n. 4, p. 518-525, 2012

BARATIERI, T.; MARCON, S. S. Longitudinalidade do cuidado: compreensão dos enfermeiros que atuam na estratégia saúde da família. Esc. Anna Nery, Rio de Janeiro, v. 15, n. 4, 2011.

BATISTA, G. S. Diretrizes da Sociedade Brasileira de Pneumologia e Tisiologia para o manejo da Asma - 2012. Residência Pediátrica, Rio de Janeiro, v. 3, n. 2, p. 47-8, 2013. Disponível em: <http://www.sbpt. org.br/downloads/arquivos/COM_ASMA/SBPT_ DIRETRIZES_MANEJO_ASMA_SBPT_2012.pdf>. Acesso em: 23 fev. 2015.

BRASIL. Ministério da Saúde. Secretaria de Atenção à Saúde. Doenças respiratórias crônicas. Caderno de Atenção Básica, Brasília, DF, n. 25. 2010. Disponível em: $<$ http://bvsms.saude.gov.br/bvs/publicacoes/doencas_ respiratorias_cronicas.pdf $>$. Acesso em: 26 maio 2014.

CARDOSO, J. P.; OLIVEIRA, M. N. D. Tendência temporal das doenças respiratórias no município de Salvador. RBPS, Fortaleza, v. 24, n. 1, p. 31-39, 2011.

CORIOLINO, M. W. L. et al. Repercussão de uma intervenção educativa com agentes comunitários de saúde nas condições ambientais de domicílios de crianças asmáticas. $J$ bras pneumol., Brasília, DF, v. 37, n. 3, 2011.

CUNHA, E. M.; GIOVANELLA, L. Longitudinalidade/ continuidade do cuidado: identificando dimensões e variáveis para a avaliação da Atenção Primária no contexto do sistema público de saúde brasileiro. Ciênc. saúde colet., Rio de Janeiro, v. 16, supl. 1, p. 1029-1042, 2011.

FELIZOLA, M. L. B. M. et al. Prevalência de asma brônquica e de sintomas a ela relacionados em escolares do Distrito Federal e sua relação com o nível socioeconômico. J bras pneumol., Brasília, DF, v. 31, n. 6 , p. 486-91, 2005.

FONTES, M. J. F. et al. Impacto de um programa de manejo da asma sobre as hospitalizações e os atendimentos de urgência. J. Pediatr. (Rio J.), Porto Alegre, v. 87, n. 5, 2011.

GIGHIO, L. A.; BARRETO, D. S.; FAJARDO, A. P. Reflexões de uma equipe de saúde e sua população adscrita sobre longitudinalidade da atenção. Rev. APS., Juiz de Fora, v. 17, n. 2, p. 244-254, 2014.

GLOBAL INITIATIVE FOR ASTHMA (GINA). Global strategy for asthma management and prevention. Institute of Heart, Lung and Blood Institute of Health. National Institute of Health Bethesda. 2010. Disponivel em: <http://ginasthma.org/>. Acesso em: 27 ago. 2014.

KINCHOKU, V. M. et al. Fatores associados ao controle da asma em pacientes pediátricos em centro de referência. Rev. Paul. Pediatr., São Paulo, v. 29, n. 4, 2011.

MARTINS, L. F. P.; MENEGHIUM, M. C., MARTINS, L. C.; PEREIRA, A. C. Avaliação da qualidade nos serviços públicos de saúde com base na percepção dos usuários e dos profissionais. RFO, Passo Fundo, v. 19, n. 2, p.151-158, 2014.

PEROSA, G. B. et al. Qualidade de vida de crianças e adolescentes asmáticos: sua relação com estratégias de enfrentamento materno. Rev. Paul. Pediatr., São Paulo, v. 31, n. $2,2013$.

ROCHA J. I. P. et al. Relação entre a ocorrência de asma em crianças com: nível educacional dos cuidadores, renda domiciliar e condições de moradia. Rev. Fac. Ciênc. Méd., Sorocaba, v. 13, n. 1, p. 17-21, 2011.

SANTOS, A. M.; GIOVANELLA, L. Estratégia saúde da família na coordenação do cuidado em região de saúde na Bahia. Saúde em Debate, Rio de Janeiro, v. 40, n. 108, p. 48-63, jan./mar. 2016. 
SOCIEDADE BRASILEIRA DE PNEUMOLOGIA E TISIOLOGIA (SBPT). Diretrizes para o manejo da asma. J bras Pneumol., Brasília, DF, v. 38, supl.1, S1-S46, 2012.

SOUZA, A. M. V.; MARCH, M. F. P. Política sanitária para asma no Brasil. Rio de Janeiro: SOPERJ, 2010.

TELLES FILHO, P. A. L. Asma brônquica. 2012.

Disponível em: <http://www.asmabronquica.com.br/ medical/epidemiologia.html>. Acesso em: 5 maio 2014.

TURRINI, R. N. T.; LEBRÃO, M. L.; CESAR, C. L. G.

Resolutividade dos serviços de saúde por inquérito domiciliar: percepção do usuário, Cad. Saúde Pública, Rio de Janeiro, v. 24, n. 3, p. 663-674, mar., 2008.

WEHRMEISTER, F. C.; PERES, K. G. A. Desigualdades regionais na prevalência de diagnóstico de asma em crianças: uma análise da Pesquisa Nacional por Amostra de Domicílios, 2003. Cad. Saúde Pública, Rio de Janeiro, v. 26, n. 9, p. 1839-1852, 2010.

Recebido para publicação em fevereiro de 2016

Versão final em agosto de 2016

Conflito de interesses: inexistente

Suporte financeiro: não houve 